\title{
Continuous subcutaneous insulin infusion versus multiple daily insulin injections in patients with diabetes mellitus: systematic review and meta-analysis
}

\author{
K. Jeitler • K. Horvath • A. Berghold • T. W. Gratzer • \\ K. Neeser • T. R. Pieber • A. Siebenhofer
}

Received: 12 September 2007 / Accepted: 5 February 2008/Published online: 20 March 2008

(C) Springer-Verlag 2008

\begin{abstract}
Aims We compared the effects of continuous subcutaneous insulin infusion (CSII) with those of multiple daily insulin (MDI) injections on glycaemic control, risk of hypoglycaemic episodes, insulin requirements and adverse events in type 1 and type 2 diabetes mellitus.

Methods The electronic databases MEDLINE, EMBASE and CENTRAL were systematically searched for randomised controlled trials up to March 2007. A systematic review and meta-analysis were performed.

Results Overall, 22 studies were included (17 on type 1 diabetes mellitus, two on type 2 diabetes mellitus, three on children). With regard to adults with type 1 diabetes mellitus, our meta-analysis found a between-treatment difference of
\end{abstract}

Electronic supplementary material The online version of this article (doi:10.1007/s00125-008-0974-3) contains supplementary material, which is available to authorised users.

K. Jeitler $(\bowtie) \cdot$ K. Horvath $\cdot$ T. W. Gratzer • T. R. Pieber

A. Siebenhofer

Diabetes and Metabolism Outpatient Clinic,

Department of Internal Medicine, Medical University of Graz,

Auenbruggerplatz 15,

8036 Graz, Austria

e-mail: klaus.jeitler@klinikum-graz.at

K. Jeitler $\cdot$ T. W. Gratzer $\cdot$ T. R. Pieber

Joanneum Research,

Institute of Medical Technologies and Health Management,

Graz, Austria

\author{
A. Berghold \\ Medical University of Graz, \\ Graz, Austria \\ K. Neeser \\ Institute for Medical Informatics and Biostatistics, \\ Basel, Switzerland
}

Institute for Medical Informatics, Statistics and Documentation,
$-0.4 \% \mathrm{HbA}_{1 \mathrm{c}}$ (six studies) in favour of CSII therapy. Available median rates of mild or overall hypoglycaemic events were comparable between the different interventions (1.9 [0.9-3.1] [CSII] vs 1.7 [1.1-3.3] [MDI] events per patient per week). Total daily insulin requirements were lower with CSII than with MDI therapy. In patients with type 2 diabetes mellitus, CSII and MDI treatment showed no statistically significant difference for $\mathrm{HbA}_{1 \mathrm{c}}$. The incidence of mild hypoglycaemic events was comparable between the treatment groups. In adolescents with type 1 diabetes mellitus, glycated haemoglobin and insulin requirements were significantly lower in the CSII groups; no data were available on hypoglycaemic events. The only study performed in younger children did not provide enough data for conclusive inferences. No overall conclusions were possible for severe hypoglycaemia and adverse events for any of the different patient groups due to rareness of such events, different definitions and insufficient reporting.

Conclusions/interpretation CSII therapy in adults and adolescents with type 1 diabetes mellitus resulted in a greater reduction of glycated haemoglobin, in adult patients without a higher rate of hypoglycaemia. No beneficial effect of CSII therapy could be detected for patients with type 2 diabetes mellitus.

Keywords Diabetes mellitus · Drug administration schedule - Glycosylated haemoglobin A · Hypoglycaemia . Insulin/administration and dosage - Insulin infusion systems . Meta-analysis · Randomised controlled trials · Review · Subcutaneous injections
Abbreviations
CSII continuous subcutaneous insulin infusion
MDI multiple daily insulin
SMD standardised mean difference
WMD weighted mean difference 


\section{Introduction}

Insulin therapy is a pharmaceutical treatment option used to lower blood glucose in diabetic patients. For insulin-treated patients blood glucose optimisation can be managed by different types of insulin and different treatment strategies such as multiple daily insulin (MDI) regimens, continuous subcutaneous insulin infusions (CSII) or conventional treatment.

CSII treatment was introduced in the 1970 s as a way of achieving and maintaining strict control of blood glucose concentrations in people with type 1 diabetes mellitus [1]. Since then numerous different insulin pumps have been developed and initial technical problems have diminished. In the last decade, devices have become much safer, smaller and more reliable. Both CSII and MDI are forms of intensified insulin treatment. However, due to its continuous basal insulin substitution, CSII can better mimic a physiological situation. Thus CSII may provide a more efficient mode of insulin delivery to the bloodstream and minimise the risk of hypoglycaemic events. It may be more beneficial to those who find it difficult to adhere to a regimen of multiple injections. On the other hand, adverse events may arise including pump malfunction, catheter infection, irritation or discomfort [2, 3]. According to some recent publications [4-6], such events now occur less often.

Recently published meta-analyses of studies comparing CSII and MDI treatment [7, 8] demonstrated an improvement in glycaemic control with the use of CSII therapy in patients with type 1 diabetes mellitus. Currently, only a few reviews are available on adults, adolescents or younger children with type 1 and patients with type 2 diabetes mellitus. To evaluate the possible advantages of CSII over MDI treatment, we performed a systematic review and meta-analysis according to the QUOROM statement [9] and based on randomised controlled trials. We considered findings on glycaemic control, insulin requirements, hypoglycaemia and adverse events.

\section{Methods}

\section{Criteria for inclusion}

For our systematic review we considered all randomised controlled trials that had been conducted with people of any age or sex suffering from type 1 or type 2 diabetes mellitus and who were on insulin treatment (excluding pregnant women). The duration of intervention had to be at least 4 weeks with a sample size of not less than ten patients. We took account of all studies that compared CSII, applied $24 \mathrm{~h} /$ day, with an MDI injections therapy (thus studies with pump therapy during the night only were excluded). Insulin therapy was classified as MDI if more than 50\% of all patients in the comparison group had three or more injections of short-acting insulin per day. The kind of short-acting insulin had to be the same in both treatment groups, either a regular insulin or an insulin analogue. Studies dealing with a mixed group of diabetic patients (type 1, type 2, children) were excluded from the review unless the respective results had been presented separately for each type of patient.

Search strategy for identification of studies

The search for randomised controlled trials was performed in MEDLINE, EMBASE and CENTRAL. The following were searched for relevant secondary literature: Cochrane Database of Systematic Reviews; Database of Abstracts of Reviews of Effects; Health Technology Assessment Database; and the National Health Service Economic Evaluation Database.

All searches were last updated on 5 March 2007. The phrases and terms used for the search in full text and subject headings, and adapted depending on the database, were: csii, continuous subcutaneous insulin, insulin pump, insulin infusion systems. We also hand-searched reference lists from relevant secondary literature.

\section{Assessed study outcomes}

We evaluated glycaemic control if expressed as a percentage of glycated haemoglobin $\left(\mathrm{HbA}_{1}\right.$ or $\left.\mathrm{HbA}_{1 \mathrm{c}}\right)$, insulin requirements, occurrence of severe and mild hypoglycaemic episodes and adverse events.

Study selection, data collection and quality assessment

Two reviewers independently evaluated the references that were thus found for relevance on the basis of their titles and abstracts. References considered as relevant by only one reviewer were reassessed by both and classified by consensus. Ultimately, a list of potentially relevant publications was prepared and full texts of these works were obtained for further evaluation. Data from each study included were extracted by the two independent reviewers. The methodological quality of each trial was assessed using a modification of the criteria given in the Cochrane Handbook [10] and by Jadad et al. [11] and Schulz et al. [12]. A list of the key domains used for our assessment is provided in the Electronic supplementary material (ESM) Table 1. We used three categories for assessment: ' $A$ ' means that plausible bias is unlikely to affect the results seriously, 'B' indicates that plausible bias raises some doubt about the results and ' $\mathrm{C}$ ' stands for plausible bias seriously weakening confidence in the results. 
Statistical analysis

The only meta-analysis performed was the analysis of glycaemic control in studies performed on adult patients with type 1 diabetes. We used a random effects model to conduct this meta-analysis for glycated haemoglobin. The standardised mean difference (SMD) was calculated for the percentage of glycated haemoglobin since different methods of measuring glycated haemoglobin were employed. The estimated combined treatment effect in absolute units was obtained by multiplying the SMD with the overall pooled estimate of $\mathrm{SD}$. For the subgroups $\mathrm{HbA}_{1 \mathrm{c}}$ and $\mathrm{HbA}_{1}$, the weighted mean differences (WMD) were calculated. The individual studies used parallel or crossover design, which we incorporated into the meta-analysis. For the crossover studies, the SEM of the within-person differences was needed to conduct the meta-analysis. In three of the seven crossover studies, the correlation between the different treatment outcomes was approximated using the lowest observed correlation among the other studies $(r=0.52)$ to calculate the SEM for the within-person differences. Heterogeneity between trials was assessed by the $\chi^{2}$ test and Higgins $I^{2}$, which describes the percentage of the variability in effect estimates that is due to heterogeneity. To explore heterogeneity, different subgroup and sensitivity analyses were performed.

\section{Results}

\section{Identification of relevant studies}

The initial search using the search strategy described above yielded 1,228 references. After exclusion of duplicates, there were 673 hits, of which 557 were classified as irrelevant by the reviewers on the basis of their abstracts. Among the remaining 116 publications that were perused in full text, 83 did not meet the inclusion criteria. For further detailed information see Fig. 1. No further full published study was obtained by screening the references of the 109 secondary literature publications retrieved. In the end, 33 publications with usable information were included in our systematic review. Of these, 17 studies (27 publications [13-39]) included mainly adult type 1 diabetic patients, three trials (four publications [40-43]) only children of up to 18 years and two studies (two publications [44, 45]) examined patients with type 2 diabetes mellitus only.

\section{Description of included trials}

Adult patients with type 1 diabetes mellitus All studies had an open-label study design. Seven of the included studies

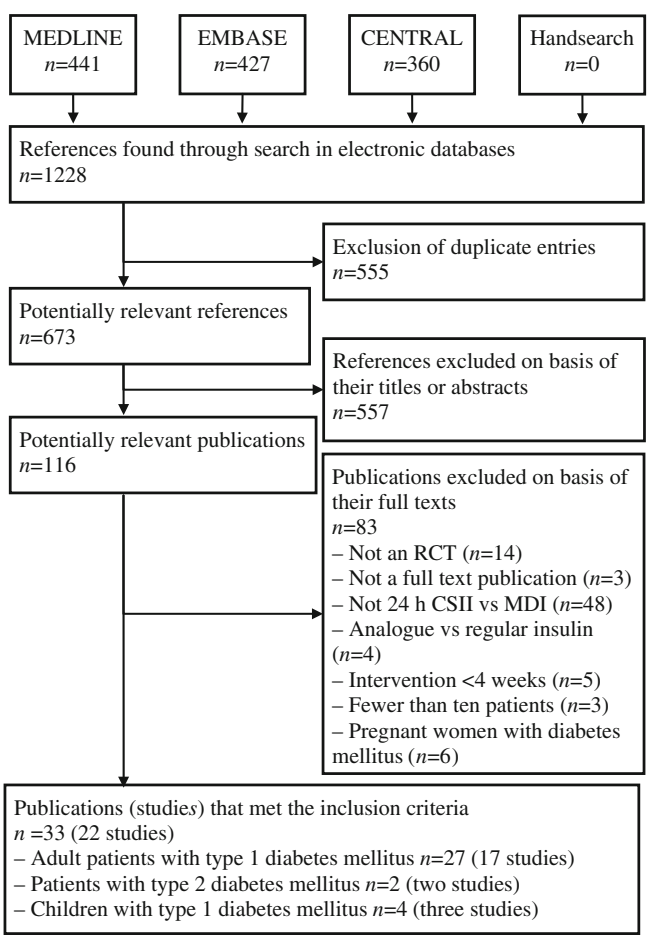

Fig. 1 Flow diagram of the literature search and identification of the studies relevant for inclusion in the systematic review. RCT, randomised controlled trial

were parallel studies and ten had a crossover design. Altogether 908 participants took part in the 17 randomised controlled studies. The study periods lasted from a minimum of 5 weeks to a maximum of 2 years. Upon our request, the authors of the Oslo Study [17, 18, 23, 25-29] told us that the study was originally intended to last for 2 years, but was later extended to 4 years. Due to incomplete data records after 4 years, only the results from the 2 year analysis were considered for this review. Twelve studies used regular human or porcine insulin and five studies used insulin analogues as short-acting insulin for CSII and MDI treatment. For further detailed information on the type of insulin and the type of pumps used, see Table 1. For further information on special study characteristics, such as indications for CSII treatment, injection rates and other relevant aspects of care, see ESM Table 2.

In one study [32], MDI treatment was compared with two different CSII regimens, one with insulin pump therapy with a basal fixed overnight insulin rate, the other with insulin pump therapy with a higher variable dawn insulin rate ( 0500 to 0800 hours). The quality of 15 studies was assessed to be in category $\mathrm{C}$ and only two studies were of higher quality (category B). One study had such a serious quality deficiency [32] that we did not include the study results in the respective analyses. In that study, patients were asked immediately after a satisfactory randomisation process ( $n=96$ patients) if they accepted their assigned 


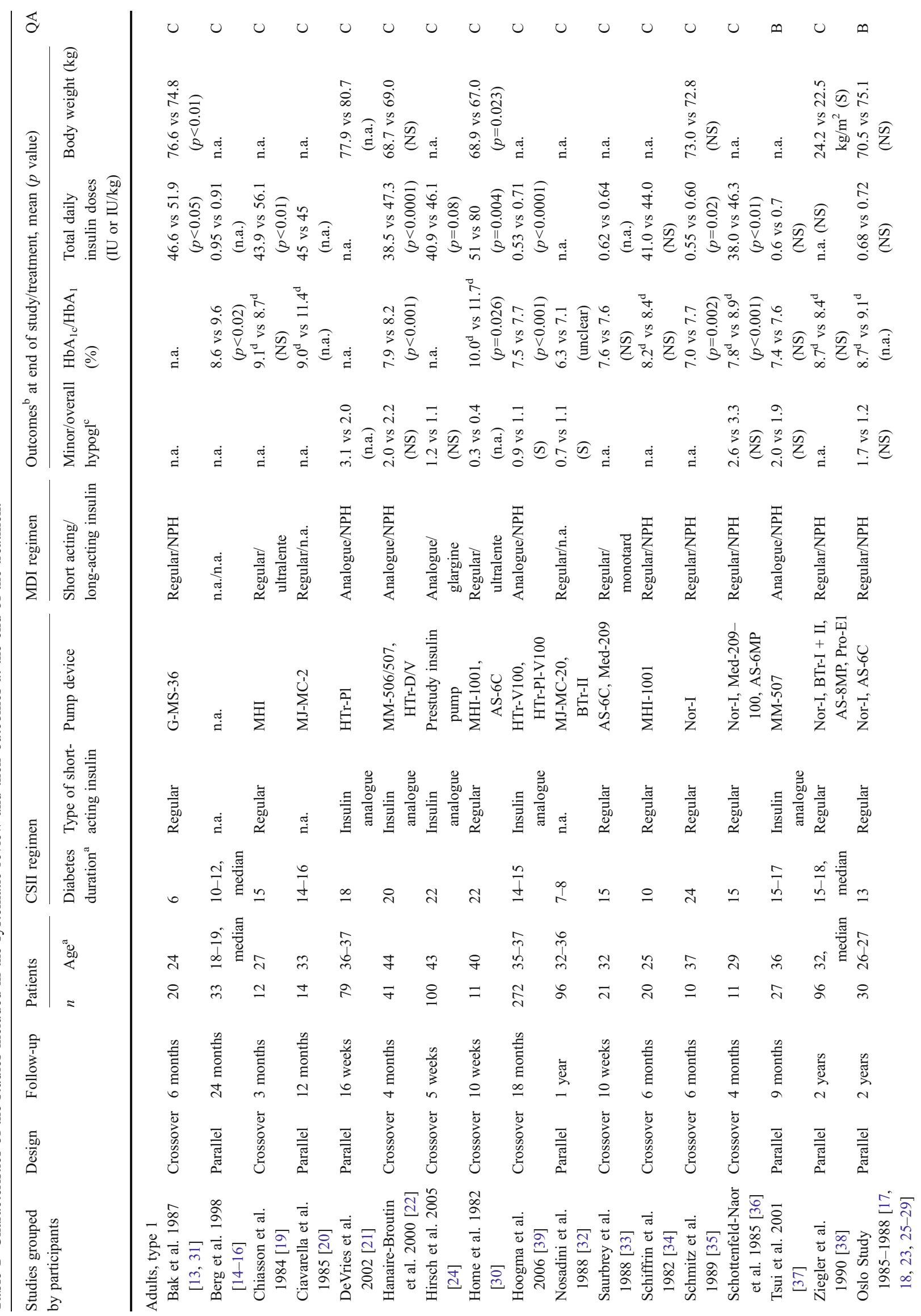


$\infty \cup \infty \cup$

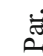

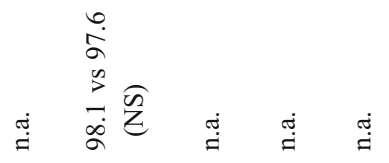

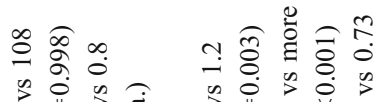

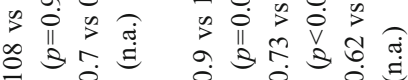

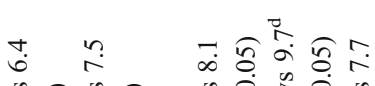

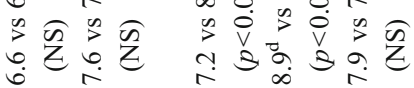

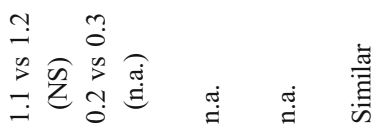

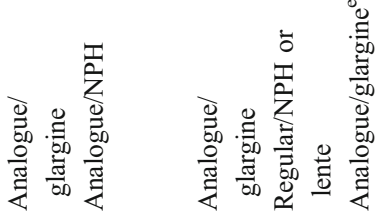

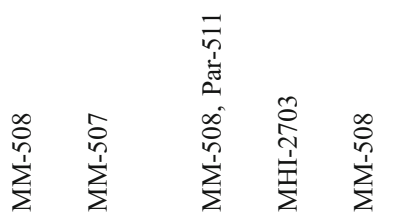

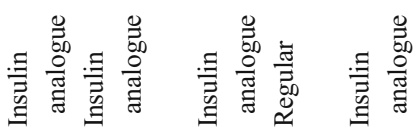

$=\frac{I}{I} \quad \hat{b} a-$

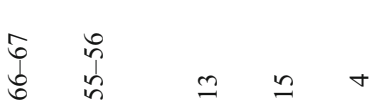

ฮ

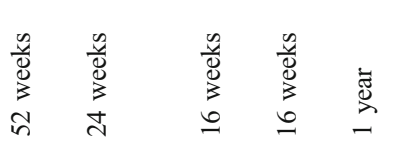

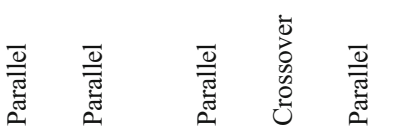

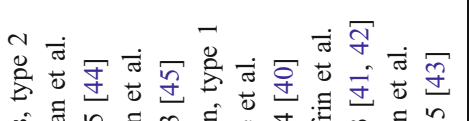

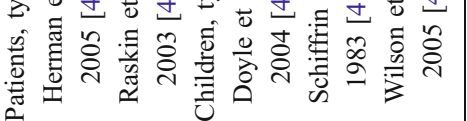

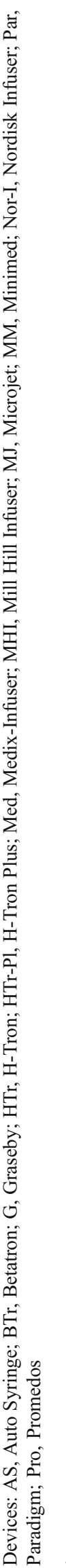


treatment. If they refused, they were excluded from the study. This is not standard procedure in trials, and in fact 32 patients in this study dropped out before even starting with the assigned treatment.

Patients with type 2 diabetes mellitus Both studies had an open-label parallel study design [44, 45] and 234 patients took part in the randomised controlled trials. The study periods were 24 and 52 weeks, respectively. Both studies used insulin analogues as short-acting insulin for CSII and MDI treatment. For further detailed information on the type of insulin and the type of pumps used, see Table 1. The quality of one study was assessed to be in category $\mathrm{C}$ and the other study was of higher quality (category B).

Children with type 1 diabetes mellitus Of the randomised controlled trials found, one [41] used a crossover design, whereas the other two, far more recent investigations [40, 43], were of parallel design. Altogether, 74 children took part in the three randomised controlled studies. The duration of follow-up and treatment was 16 weeks in two trials and 52 weeks in the third trial. Two studies were performed in adolescents [40, 41]. One study [43] included young children with a mean age of 4 years. In the older study [41], regular insulin was used; in both recent studies $[40,43]$ insulin analogues were used as pump insulin and as bolus insulin in the respective comparison groups. Insulin glargine was used as basal insulin in the MDI groups by all patients in one trial [40] and by $60 \%$ of patients at the end of the other study [43], while the other patients in that trial used NPH and ultralente as basal insulin. The quality of one study was assessed to be in category $\mathrm{C}$ and the other two studies were of higher quality (category B).

Glycaemic control—glycated haemoglobin

Adult patients with type 1 diabetes mellitus - meta-analysis Of 17 selected studies we had to exclude five studies from this meta-analysis for the following reasons: (1) one study did not report any $\mathrm{HbA}_{1 \mathrm{c}}$ baseline and follow-up data [13]; (2) one study compared only treatment sequences (CSII-MDI vs MDI-CSII) at study endpoint [24]; (3) another trial provided no measure of variability [37]; (4) in one study [36] the discrepancies in the measurement of variability could not be resolved; and (5) another study [32] had an unacceptably high dropout rate immediately after randomisation assignment.

Ultimately, 12 studies were included in the meta-analysis of the percentage of glycated haemoglobin at the end of treatment. When the SMD was calculated, the effect between treatment groups was estimated to be -0.6 (95\% CI: -0.87 , -0.22 ) in favour of CSII compared with MDI (Fig. 2), which corresponds to an effect size of $-0.6 \%$ in original units. The $I^{2}$ statistic was estimated to be $75 \%$, which indicates substantial heterogeneity.

Six of the twelve studies used $\mathrm{HbA}_{1 \mathrm{c}}$, whereas the other six studies used $\mathrm{HbA}_{1}$ for the measurement of glycated haemoglobin. Analyses according to the different methods showed that for studies using $\mathrm{HbA}_{1 \mathrm{c}}$ the difference between the CSII and MDI groups was statistically significant in favour of CSII treatment with a WMD of $-0.4 \%(95 \% \mathrm{CI}$ : $-0.65,-0.20 ; I^{2}=72 \%$ ) (Fig. 3a). For the studies in which $\mathrm{HbA}_{1}$ was measured, the WMD was $-0.6 \%(95 \% \mathrm{CI}$ : $\left.-1.34,0.14 ; I^{2}=84 \%\right)$. The difference between the groups was not statistically significant (Fig. 3b).

In studies with a duration of less than 6 months, the SMD in glycated haemoglobin when comparing CSII with MDI treatment was $-0.4(95 \% \mathrm{CI}:-0.82,-0.01)$, which is equivalent to $-0.4 \%$ in original units. In studies of longterm duration (6 months or more) comparing CSII with MDI treatment, the SMD was -0.7 (95\% CI: $-1.24,-0.19)$, which corresponds to $-0.8 \%$ in original units. In both analyses, there was evidence of heterogeneity among short $\left(I^{2}=55 \%\right)$ and long-term studies $\left(I^{2}=82 \%\right)$. For studies using a parallel design, the SMD in glycated haemoglobin was -0.9 (95\% CI: $-1.64,-0.10 ; I^{2}=85 \%$ ) when comparing CSII and MDI treatment, corresponding to $-1.2 \%$ in original units. For studies with crossover design the SMD in glycated haemoglobin was -0.4 (95\% CI: $-0.68,-0.07$; $I^{2}=52 \%$ ), corresponding to $-0.4 \%$ in original units. For
Fig. 2 Glycated haemoglobin at the end of treatment in the studies performed on adult patients with type 1 diabetes: standardised mean difference (SMD) between groups. Model with random effects: DerSimonian-Laird. Heterogeneity: $Q=$ 43.16, $d f=11(p=0.000), I^{2}=$ $74.5 \%$. Overall effect: $z$ score $=$ $-3.31(p=0.001), \tau^{2}=0.212$

\begin{tabular}{l} 
Study \\
\hline Berg et al., 1998 [14-16] \\
Ciavarella et al., 1985 [20] \\
DeVries et al., 2002 [21] \\
Ziegler et al., 1990 [38] \\
Oslo Study, 1985-1988 [17, 18, 23, 25-29] \\
Chiasson et al., 1984 [19] \\
Hanaire-Broutin et al., 2000 [22] \\
Home et al., 1982 [30] \\
Hoogma et al., 2006 [39] \\
Saurbrey et al., 1988 [33] \\
Schiffrin et al., 1982 [34] \\
Schmitz et al., 1989 [35] \\
Total (95\% Cl)
\end{tabular}

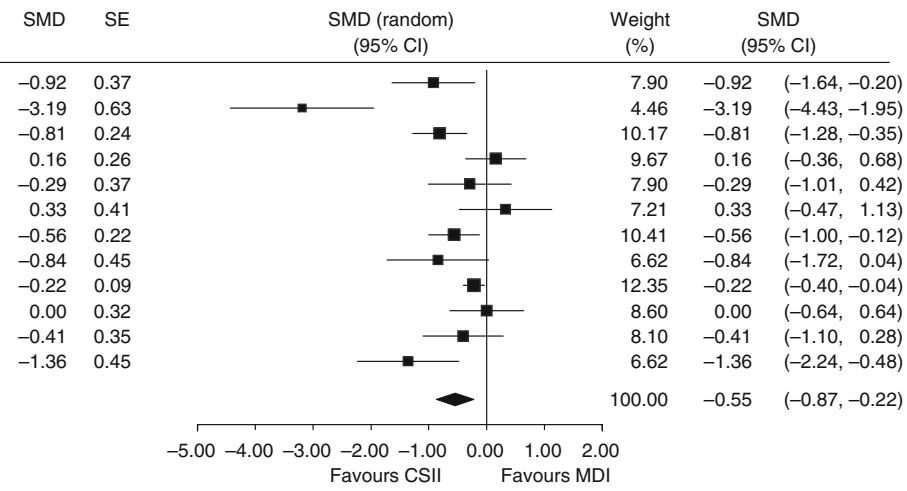


Fig. 3 a, b Glycated haemoglobin at the end of treatment in the studies performed on adult patients with type 1 diabetes: weighted mean difference (WMD) between groups analysed by method of measurement. a Studies using glycated effects: DerSimonian-Laird. Heterogeneity: $Q=17.77, d f=5$ $(p=0.003), I^{2}=71.9 \%$. Overall effect: $z$ score $=-3.7(p=0.000)$, $\tau^{2}=0.048$. b Studies using glycated $\mathrm{HbA}_{1}$. Model with random effects: DerSimonian-Laird. Heterogeneity: $Q=30.34, d f=5$ $(p=0.000), I^{2}=83.5 \%$. Overall effect: $z$ score $=-1.59(p=$ $0.113), \tau^{2}=0.659$ $\mathrm{HbA}_{1 \mathrm{c}}$. Model with random

\section{a}

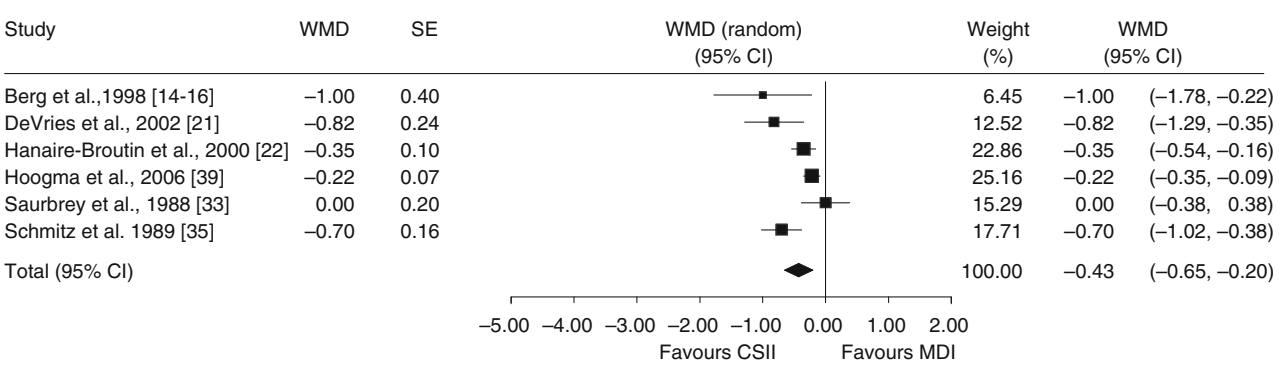

b

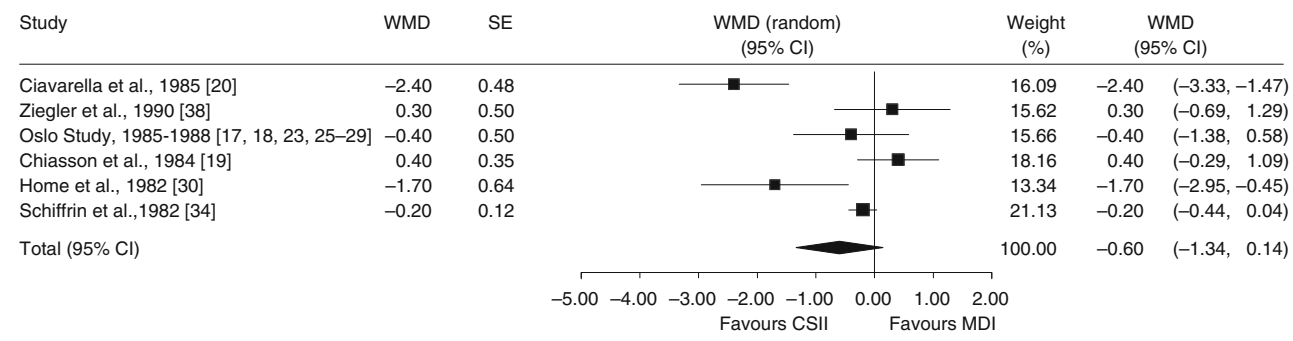

studies published in 2000 or later, the WMD was $-0.4 \%$ (95\% CI: $-0.60,-0.14 ; I^{2}=69 \%$ ) when comparing CSII and MDI treatment.

Patients with type 2 diabetes mellitus In both trials [44, 45] no significant differences in $\mathrm{HbA}_{1 \mathrm{c}}$ between the two treatment groups were found at the end of the study (Table 1).

Children with type 1 diabetes mellitus Glycated haemoglobin decreased more pronouncedly in patients treated with CSII in all three included trials. At the end of treatment $\mathrm{HbA}_{1 \mathrm{c}} / \mathrm{HbA}_{1}$ was significantly lower in the CSII group in each of the trials performed in adolescents [40, 41]. In the trial including young children [43], glycated haemoglobin was slightly higher in CSII-treated patients but without reaching statistical significance (Table 1).

Total insulin requirements

Adult patients with type 1 diabetes mellitus Of 14 studies reporting total insulin requirements at the end of treatment, $12[13,19,22,24,28,30,33-37,39]$ consistently found that the insulin doses were lower in CSII-treated patients. In seven $[13,19,22,30,35,36,39]$ of these investigations, the difference was statistically significant.

One study [20] did not find any difference between the treatment groups and another [16] showed insulin requirements to be higher under pump therapy, but without statistical significance. In the latter trials, patients randomised to CSII treatment started treatment with a higher insulin dosage and could either reduce it more efficiently or showed a smaller increase in required insulin doses during the study period than patients in the MDI groups.

Patients with type 2 diabetes mellitus There were no reported significant differences in insulin requirements at the end of studies. Raskin et al. [45] showed a slight reduction in insulin requirements in the CSII group compared with the MDI group, while Herman et al. [44] did not find any difference at all.

Children with type 1 diabetes mellitus At the end of treatment, all three studies reported total insulin requirements to be lower in the CSII group than in the MDI group, which in the two studies with adolescents [40, 41] was statistically significant.

\section{Hypoglycaemia}

Divergence between studies Due to differences in the definitions of hypoglycaemic episodes and in the study duration ( 5 to 104 weeks), and because of the often missing measures of variance and the small numbers of patients with severe hypoglycaemic episodes, no meta-analysis of hypoglycaemic events was performed.

Severe hypoglycaemia, adult patients with type 1 diabetes mellitus All in all, severe hypoglycaemic episodes were rare. Four of 17 studies [16, 19, 20, 30] did not mention severe hypoglycaemic episodes. Three others $[33,35,36]$ reported that no severe hypoglycaemia was observed. Information on the number of patients with severe hypoglycaemic episodes 
could be found in six studies [13, 21, 22, 24, 28, 34] and four [32, 37-39] at least reported on the rates or the number of severe hypoglycaemic events in the treatment groups. The proportion of patients with severe hypoglycaemic episodes ranged from 0 to 0.13 in the CSII group and from 0 to 0.4 in the MDI group. Only two studies [21,28] reported a formal statistical test result for the number of patients with severe hypoglycaemic episodes in the treatment groups, which in both studies did not differ significantly.

Mild/minor/symptomatic hypoglycaemia in adult patients with type 1 diabetes mellitus In six of 17 studies [13, 16, $19,20,33,35]$ there were no reports on mild, minor or symptomatic hypoglycaemic events. Two other studies [30, 37] presented results for overall hypoglycaemia only, but for the latter [37] the rate of mild/minor hypoglycaemic events could be calculated because exact numbers of severe events were available. Six studies [21, 22, 24, 28, 36, 39] provided the event rates for mild/minor hypoglycaemia per patient per time period, which ranged from 0.9 to 3.1 weekly events per patient in the CSII groups and from 1.1 to 3.3 in the MDI groups (ranges include previously mentioned study [37]). The median rate per patient per week was 1.9 in the CSII groups and 1.7 in the MDI groups. Due to its high dropout rate one further study [32] that also reported event rates per patient per time was not considered in the above summary. One study [34] presented only the total number of mild and moderate events for treatment. The 2 year trial by Ziegler [38] reported just the rate of symptomatic events per group for 6 month periods; it was always higher in the CSII group and statistically significant in three of these periods.

Severe hypoglycaemia, patients with type 2 diabetes mellitus Information on severe hypoglycaemia is provided in both publications. All in all, severe hypoglycaemic episodes were rare. In one study [45], there were no severe hypoglycaemic episodes and in the other study [44] three patients in the CSII compared with six patients in the MDI group experienced severe hypoglycaemia. The event rate per patient-year was not significantly different, although it tended towards a reduction in favour of CSII treatment (CSII, 0.1 vs MDI, 0.2 events per patient-year).

Mild hypoglycaemia, patients with type 2 diabetes mellitus Detailed data for the occurrence of mild hypoglycaemic episodes are given in both studies included here [44, 45], with a non-significant reduction of frequency in the CSII group in the former [44] (CSII, 1.1 vs MDI, 1.2 events per patient per week). In Raskin [45] the event rate was also lower in the CSII group, with 0.8 vs 1.2 events per patient per 30 days in the MDI-treated group. But a formal statistical test was not presented.
Severe hypoglycaemia, children with type 1 diabetes mellitus In all included trials, the number of severe hypoglycaemic events was small. Only three severe hypoglycaemic events were recorded for patients in the CSII groups and six events in the MDI groups in all trials taken together. In one investigation [40], four patients in the MDI group versus one in the CSII group experienced each exactly one severe hypoglycaemic event. No difference was found for CSII and MDI treatment in the other two trials, with one event in each group in both studies [41, 43], respectively.

No information on mild/minor/symptomatic hypoglycaemia in children with type 1 diabetes mellitus was available.

\section{Adverse events}

Adult patients with type 1 diabetes mellitus Overall, information on adverse treatment effects other than hypoglycaemia was insufficient in the available publications.

Only four studies reported on serious adverse events besides hypoglycaemia. The rates of occurrence were generally low and in two studies $[35,36]$ there were no serious adverse events. In another study [38] only one serious adverse event was reported as causing a dropout in the CSII group. Another team [39] reported 15 serious adverse events in the MDI group compared with 20 events in the CSII group (including four events of ketoacidosis).

Data on ketoacidosis were given in ten studies. Only one of these [32] reported a statistically significant increase in the occurrence of ketoacidosis in the MDI group compared with both CSII groups (fixed and variable infusion rate), but this study was not considered due to major quality deficiencies after the randomisation process. Ziegler et al. [38] reported data on ketoacidosis indicating that the events per 100 patient-years were higher in the CSII group (intention to treat and per protocol analysis), but the difference was not statistically significant. All the other studies noted only a few ketotic events: in three studies there was one event in the CSII group only [24, 33, 36], another [21] reported one event in both treatment arms, yet another [39] reported four events in the CSII and none in the MDI group and the Oslo trial [28] found that two patients in the CSII vs no patients in the MDI group developed ketoacidosis. Data on hyperglycaemia were given in only one publication included in this review. However, due to major quality deficiencies, the result of hyperglycaemic episodes in that study [32] will not be considered for interpretation.

Four studies reported on infusion site problems, all with an increased rate in the CSII group. Hoogma [39] found that $8.2 \%$ of the patients in the CSII vs $0.8 \%$ in the MDI group had problems at the infusion site, without giving more detailed information. In another investigation [33], 
three episodes of subcutaneous infection (one requiring surgical incision) occurred in the same patient in the CSII group, who also had ketoacidosis. Elsewhere [36], infusion site problems were noted under pump therapy only twice and several reasons for infusion problems were reported, i.e. catheter problems, pump arrest or dosage errors, battery problems, syringe dislocation. The Oslo Study [28] reported that eight events in six patients in the CSII vs none in the MDI group involved subcutaneous abscesses. Only one publication [32] included information on mortality, reporting that in the CSII group with variable infusion rates, one patient died. This patient had presented with high blood glucose values the last 2 days before his death, he suffered from fever and acetonuria, and his blood glucose values had not responded to an increased insulin infusion rate.

Patients with type 2 diabetes mellitus Both publications provided information on local reactions at the injection site. In one [44] significantly more patients in the MDI group reported bleeding or bruising events as compared with the CSII group, where significantly more patients had infusion site inflammation or irritation. In the other study [45] only CSII-treated patients reported injection site reactions, but no data on significance were given. Both teams found that technical problems were more common in the respective CSII groups than in the MDI groups. Data on hyperglycaemic episodes were given in one publication [45] included in this review. These episodes occurred considerably more frequently in MDI patients, but nothing was said about possible statistical significance.

Children with type 1 diabetes mellitus Doyle [40] reported one episode of ketoacidosis for each of the two treatment groups, while the other study [43] reported no event for any of the groups. There is no more information on any other adverse events in any of the publications.

\section{Discussion}

Based on the findings of this systematic review and metaanalysis, the following conclusions can be drawn on CSII therapy in patients with type 1 and type 2 diabetes mellitus.

In adult patients with type 1 diabetes mellitus, CSII treatment in comparison to MDI therapy leads to better glycaemic control without a rise in hypoglycaemic events and with lower insulin requirements. CSII can thus be considered a valuable therapy option in adult patients with type 1 diabetes mellitus. In patients with type 2 diabetes mellitus treatment with CSII did not result in better glycaemic control when compared with MDI therapy. Also there was no difference in hypoglycaemic events and insulin requirements between the two treatments. It can therefore be concluded that in patients with type 2 diabetes mellitus CSII application is not superior to MDI treatment. Results from studies investigating adolescent patients with type 1 diabetes mellitus suggested a beneficial effect of CSII therapy compared with MDI therapy in terms of glycated haemoglobin, but because of the small number of patients under investigation and the short duration of the included trials, conclusions on adolescent patients are less firm than for adult patients with type 1 diabetes mellitus. There is also no clear information about hypoglycaemia in adolescents because of the insufficient reporting of such events. Due to the very limited data available, no meaningful inferences could be drawn on the possible effects of CSII treatment in younger children.

Even though this review, bringing together evidence for three important patient groups, is, to our knowledge, one of the most comprehensive on the topic to date, there are some limitations warranting caution in interpreting the validity of the results. Thus study and publication quality was low and information on features that high-quality studies would normally include were mostly missing. Furthermore, in the meta-analysis of differences in $\mathrm{HbA}_{1 \mathrm{c}}$ in patients with type 1 diabetes mellitus, a high degree of heterogeneity was detected. To investigate this heterogeneity several sensitivity analyses were performed. The positive effect of CSII treatment was likewise seen in trials that lasted for less than or more than 6 months. Comparing the studies according to their design, the result remained statistically significant in favour of CSII treatment in both parallel and crossover trials. While in studies using $\mathrm{HbA}_{1 \mathrm{c}}$ measurements CSII treatment resulted in a statistically significant lower $\mathrm{HbA}_{1 \mathrm{c}}$ value, in (the older) studies using $\mathrm{HbA}_{1}$ to measure glycaemic control the difference between the treatment groups was not statistically significant. The three studies published in the year 2000 and later, where newer devices were in use (which we also offer in our outpatient clinic at the Medical University Graz), further supported the superiority of CSII treatment. In addition, even in these studies, where short-acting insulin analogues, today probably the most commonly used bolus insulin type, were used, clear clinically relevant superiority was unproven [46].

However, none of these sensitivity analyses could explain the observed heterogeneity, which is most probably caused by a combination of several confounding factors (studies dating back to 1982, low study quality, different study designs, small treatment groups, different CSII pumps, etc.). It should also be noted that the heterogeneity is not so much caused by qualitatively different results, but by quantitative differences. From these limitations it is clear that the results obtained from this meta-analysis have to be interpreted cautiously even though they are consistent with previously published meta-analyses [7, 8, 47, 48]. 
In terms of insulin requirements, no meta-analysis was done because, from our point of view, insulin requirement is only a relevant outcome for the healthcare system but not relevant for the individual patient. In any case, a metaanalysis would not have helped interpret the data more effectively, due to differences in reporting, e.g. either as total insulin requirement per person or per body weight per day, and to the sometimes missing measures for calculation.

A further problem in interpreting the results arises from the fact that in studies in which neither the patients nor the carers are blinded to the treatment the reliability of computed results for hypoglycaemic events greatly depends on whether the definition of hypoglycaemia leaves room for interpretation influenced by subjective, voluntary or involuntary factors. Since in many of the included trials the different definitions of hypoglycaemia were susceptible to bias and the endpoint detection was not blinded, results on hypoglycaemic events have to be viewed as inherently uncertain. Like Pickup et al. [7], Colquitt et al. [47] and Weissberg-Benchell et al. [48], we also did not perform a meta-analysis on hypoglycaemic events, due to differences in definitions of hypoglycaemic episodes, the difference in study duration, the small numbers of patients with severe hypoglycaemic episodes and the often missing measures of variance.

Data on undesirable events were unsatisfactory in all studies considered for this review. The available results indicate that there was no difference in serious adverse events between groups. Ketoacidosis was somewhat more frequent in the CSII groups and the rate of local reactions and technical problems was reported to be higher in patients treated with CSII as well. One study showed a significant increase in hyperglycaemic events in the CSII group, and in another trial on patients with type 1 diabetes mellitus one death occurred in the CSII group.

While from the results of this comprehensive systematic review the effects of CSII therapy compared with MDI treatment in adult patients with type 1 diabetes mellitus and in patients with type 2 diabetes mellitus can be considered to be clear, further research is needed on the effects of CSII therapy in adolescents and younger children with type 1 diabetes mellitus.

Acknowledgements We thank M. Korsatko, S. Korsatko, G. Köhler, A. Plasnik, U. Püringer and B. Semlitsch for assistance in reviewing and collecting the data and E. Lamont for helping prepare the manuscript. We thank M. Majerowicz for the translation of the Polish papers. The sponsor of the study, Roche Diagnostics, had no role in the study design, in data collection and analysis, data interpretation or writing of the report.

Duality of interest K. Jeitler and T. W. Gratzer are employees of Joanneum Research, which received grants from Roche Diagnostics for performing systematic reviews. K. Jeitler, A. Siebenhofer, K.
Horvath and T. R. Pieber performed clinical trials with short and longacting insulin analogues and insulin pumps for Aventis, Eli Lilly, Novo Nordisk and Roche. T. R. Pieber was or is a currently paid consultant for these companies and is also on the advisory board of Novo Nordisk. K. Neeser is an employee of the Institute of Medical Informatics and Biostatistics (IMIB) in Basel, Switzerland. IMIB received grants from Roche Diagnostics for performing health economic analyses and health technology assessments.

\section{References}

1. Pickup JC, Keen H, Parsons JA, Alberti KG (1978) Continuous subcutaneous insulin infusion: an approach to achieving normoglycaemia. BMJ 1:204-207

2. Chantelau E, Spraul M, Muhlhauser I, Gause R, Berger M (1989) Long-term safety, efficacy and side-effects of continuous subcutaneous insulin infusion treatment for type 1 (insulin-dependent) diabetes mellitus: a one centre experience. Diabetologia 32:421-426

3. Guinn TS, Bailey GJ, Mecklenburg RS (1988) Factors related to discontinuation of continuous subcutaneous insulin-infusion therapy. Diabetes Care 11:46-51

4. Guilhem I, Leguerrier AM, Lecordier F, Poirier JY, Maugendre D (2006) Technical risks with subcutaneous insulin infusion. Diabetes Metab 32:279-284

5. Plotnick LP, Clark LM, Brancati FL, Erlinger T (2003) Safety and effectiveness of insulin pump therapy in children and adolescents with type 1 diabetes. Diabetes Care 26:1142-1146

6. Richardson T, Kerr D (2003) Skin-related complications of insulin therapy: epidemiology and emerging management strategies. Am J Clin Dermatol 4:661-667

7. Pickup J, Mattock M, Kerry S (2002) Glycaemic control with continuous subcutaneous insulin infusion compared with intensive insulin injections in patients with type 1 diabetes: meta-analysis of randomised controlled trials. BMJ 324:705

8. Retnakaran R, Hochman J, DeVries JH et al (2004) Continuous subcutaneous insulin infusion versus multiple daily injections: the impact of baseline A1c. Diabetes Care 27:2590-2596

9. Moher D, Cook DJ, Eastwood S, Olkin I, Rennie D, Stroup DF (1999) Improving the quality of reports of meta-analyses of randomised controlled trials: the QUOROM statement. Quality of reporting of meta-analyses. Lancet 354:1896-1900

10. Higgins JPT, Green S (eds) Cochrane handbook for systematic reviews of interventions 4.2.4 [updated March 2005]. In: The Cochrane Library (Issue 2) 2005. Wiley, Chichester

11. Jadad AR, Moore RA, Carroll D et al (1996) Assessing the quality of reports of randomized clinical trials: is blinding necessary? Control Clin Trials 17:1-12

12. Schulz KF, Chalmers I, Hayes RJ, Altman DG (1995) Empirical evidence of bias. Dimensions of methodological quality associated with estimates of treatment effects in controlled trials. JAMA 273:408-412

13. Bak JF, Nielsen OH, Pedersen O, Beck-Nielsen H (1987) Multiple insulin injections using a pen injector versus insulin pump treatment in young diabetic patients. Diabetes Res 6:155-158

14. Bangstad HJ, Kofoed-Enevoldsen A, Dahl-Jorgensen K, Hanssen KF (1992) Glomerular charge selectivity and the influence of improved blood glucose control in type 1 (insulin-dependent) diabetic patients with microalbuminuria. Diabetologia 35:1165-1169

15. Bangstad HJ, Osterby R, Dahl-Jorgensen K, Berg KJ, Hartmann A, Hanssen KF (1994) Improvement of blood glucose control in IDDM patients retards the progression of morphological changes in early diabetic nephropathy. Diabetologia 37:483-490 
16. Berg TJ, Nourooz-Zadeh J, Wolff SP, Tritschler HJ, Bangstad HJ, Hanssen KF (1998) Hydroperoxides in plasma are reduced by intensified insulin treatment. A randomized controlled study of IDDM patients with microalbuminuria. Diabetes Care 21:1295-1300

17. Brinchmann-Hansen O, Dahl-Jorgensen K, Hanssen KF, Sandvik L (1985) Effects of intensified insulin treatment on various lesions of diabetic retinopathy. Am J Ophthalmol 100:644-653

18. Brinchmann-Hansen O, Dahl-Jorgensen K, Hanssen KF, Sandvik L (1988) Oscillatory potentials, macular recovery time, and diabetic retinopathy through 3 years of intensified insulin treatment. Ophthalmology 95:1358-1366

19. Chiasson JL, Ducros F, Poliquin-Hamet M, Lopez D, Lecavalier L, Hamet P (1984) Continuous subcutaneous insulin infusion (Mill-Hill Infuser) versus multiple injections (Medi-Jector) in the treatment of insulin-dependent diabetes mellitus and the effect of metabolic control on microangiopathy. Diabetes Care 7:331-337

20. Ciavarella A, Vannini P, Flammini M, Bacci L, Forlani G, Borgnino LC (1985) Effect of long-term near-normoglycemia on the progression of diabetic nephropathy. Diabetes Metab 11:3-8

21. DeVries JH, Snoek FJ, Kostense PJ, Masurel N, Heine RJ, Dutch Insulin Pump Study Group (2002) A randomized trial of continuous subcutaneous insulin infusion and intensive injection therapy in type 1 diabetes for patients with long-standing poor glycemic control. Diabetes Care 25:2074-2080

22. Hanaire-Broutin H, Melki V, Bessieres-Lacombe S, Tauber JP (2000) Comparison of continuous subcutaneous insulin infusion and multiple daily injection regimens using insulin lispro in type 1 diabetic patients on intensified treatment: a randomized study. The Study Group for the Development of Pump Therapy in Diabetes. Diabetes Care 23:1232-1235

23. Hanssen KF, Dahl-Jorgensen K, Brinchmann-Hansen O (1985) The influence of strict control on diabetic complications. Acta Endocrinol 272:57-60

24. Hirsch IB, Bode BW, Garg S et al (2005) Continuous subcutaneous insulin infusion (CSII) of insulin aspart versus multiple daily injection of insulin aspart/insulin glargine in type 1 diabetic patients previously treated with CSII. Diabetes Care 28:533-538

25. Dahl-Jorgensen K, Brinchmann-Hansen O, Hanssen KF, Sandvik L, Aagenaes O (1985) Rapid tightening of blood glucose control leads to transient deterioration of retinopathy in insulin dependent diabetes mellitus: the Oslo study. BMJ 290:811-815

26. Dahl-Jorgensen K, Brinchmann-Hansen O, Hanssen KF et al (1986) Effect of near normoglycaemia for two years on progression of early diabetic retinopathy, nephropathy, and neuropathy: the Oslo study. BMJ 293:1195-1199

27. Dahl-Jorgensen K, Torjesen P, Hanssen KF, Sandvik L, Aagenaes O (1987) Increase in insulin antibodies during continuous subcutaneous insulin infusion and multiple-injection therapy in contrast to conventional treatment. Diabetes $36: 1-5$

28. Dahl-Jorgensen K (1987) Near-normoglycemia and late diabetic complications. The Oslo Study. Acta Endocrinol 284:1-38

29. Dahl-Jorgensen K, Hanssen KF, Kierulf P, Bjoro T, Sandvik L, Aagenaes O (1988) Reduction of urinary albumin excretion after 4 years of continuous subcutaneous insulin infusion in insulindependent diabetes mellitus. The Oslo Study. Acta Endocrinol 117:19-25

30. Home PD, Capaldo B, Burrin JM, Worth R, Alberti KG (1982) A crossover comparison of continuous subcutaneous insulin infusion (CSII) against multiple insulin injections in insulin-dependent diabetic subjects: improved control with CSII. Diabetes Care 5:466-471

31. Husted SE, Nielsen HK, Bak JF, Beck-Nielsen H (1989) Antithrombin III activity, von Willebrand factor antigen and platelet function in young diabetic patients treated with multiple insulin injections versus insulin pump treatment. Eur J Clin Invest 19:90-94
32. Nosadini R, Velussi M, Fioretto P et al (1988) Frequency of hypoglycaemic and hyperglycaemic-ketotic episodes during conventional and subcutaneous continuous insulin infusion therapy in IDDM. Diabetes Nutr Metab 1:289-296

33. Saurbrey N, Arnold-Larsen S, Moller-Jensen B, Kuhl C (1988) Comparison of continuous subcutaneous insulin infusion with multiple insulin injections using the NovoPen. Diabet Med 5:150-153

34. Schiffrin A, Belmonte MM (1982) Comparison between continuous subcutaneous insulin infusion and multiple injections of insulin. A one-year prospective study. Diabetes 31:255-264

35. Schmitz A, Christiansen JS, Christensen CK, Hermansen K, Mogensen CE (1989) Effect of pump versus pen treatment on glycaemic control and kidney function in long-term uncomplicated insulin-dependent diabetes mellitus (IDDM). Dan Med Bull $36: 176-178$

36. Schottenfeld-Naor Y, Galatzer A, Karp M, Josefsberg Z, Laron Z (1985) Comparison of metabolic and psychological parameters during continuous subcutaneous insulin infusion and intensified conventional insulin treatment in type I diabetic patients. Isr J Med Sci 21:822-828

37. Tsui E, Barnie A, Ross S, Parkes R, Zinman B (2001) Intensive insulin therapy with insulin lispro: a randomized trial of continuous subcutaneous insulin infusion versus multiple daily insulin injection. Diabetes Care 24:1722-1727

38. Ziegler D, Dannehl K, Koschinsky T, Toeller M, Gries FA (1990) Comparison of continuous subcutaneous insulin infusion and intensified conventional therapy in the treatment of type I diabetes: a two-year randomized study. Diabetes Nutr Metab 3:203-213

39. Hoogma RP, Hammond PJ, Gomis R et al (2006) Comparison of the effects of continuous subcutaneous insulin infusion (CSII) and NPH-based multiple daily insulin injections (MDI) on glycaemic control and quality of life: results of the 5-nations trial. Diab Med 23:141-147

40. Doyle EA, Weinzimer SA, Steffen AT, Ahern JA, Vincent M, Tamborlane WV (2004) A randomized, prospective trial comparing the efficacy of continuous subcutaneous insulin infusion with multiple daily injections using insulin glargine. Diabetes Care 27:1554-1558

41. Schiffrin A, Desrosiers M, Moffatt M, Belmonte MM (1983) Feasibility of strict diabetes control in insulin-dependent diabetic adolescents. J Pediatr 103:522-527

42. Schiffrin AD, Desrosiers M, Aleyassine H, Belmonte MM (1984) Intensified insulin therapy in the type I diabetic adolescent: a controlled trial. Diabetes Care 7:107-113

43. Wilson DM, Buckingham BA, Kunselman EL, Sullivan MM, Paguntalan HU, Gitelman SE (2005) A two-center randomized controlled feasibility trial of insulin pump therapy in young children with diabetes. Diabetes Care 28:15-19

44. Herman WH, Ilag LL, Johnson SL et al (2005) A clinical trial of continuous subcutaneous insulin infusion versus multiple daily injections in older adults with type 2 diabetes. Diabetes Care 28:1568-1573

45. Raskin P, Bode BW, Marks JB et al (2003) Continuous subcutaneous insulin infusion and multiple daily injection therapy are equally effective in type 2 diabetes: a randomized, parallelgroup, 24-week study. Diabetes Care 26:2598-2603

46. Siebenhofer A, Plank J, Berghold A et al (2006) Short acting insulin analogues versus regular human insulin in patients with diabetes mellitus. Cochrane Database Syst Rev, Issue 2, Art. no.: CD003287. DOI 10.1002/14651858.CD003287.pub4

47. Colquitt JL, Green C, Sidhu MK, Hartwell D, Waugh N (2004) Clinical and cost-effectiveness of continuous subcutaneous insulin infusion for diabetes. Health Technol Assess 8:1-171

48. Weissberg-Benchell J, Antisdel-Lomaglio J, Seshadri R (2003) Insulin pump therapy: a meta-analysis. Diabetes Care 26:1079-1087 